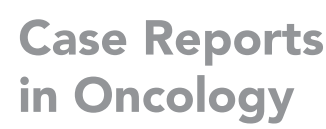

Case Reports

in Oncology

\title{
A Rare Case of Sensory Neuropathy Associated with Transitional Cell Carcinoma of the Bladder
}

\author{
Sujitha Ketinenia Sreenath Kodalib Sasikanth Gorantlac \\ aDepartment of Internal Medicine, University of Illinois College of Medicine at Peoria, \\ Peoria, IL, USA; ${ }^{b}$ Department of Hematology and Oncology, McFarland Clinic, Ames, IA, \\ USA; 'Department of Neurology, University of Illinois College of Medicine at Peoria and OSF \\ HealthCare Illinois Neurological Institute, Peoria, IL, USA
}

\section{Keywords}

Anti-Hu antibody · Paraneoplastic neurological syndrome - Transitional cell carcinoma of the bladder

\begin{abstract}
Malignancies can trigger an autoimmune response against the nervous system and manifest as paraneoplastic neurological syndromes (PNS). Initial symptoms of PNS may develop up to 5 years prior to the diagnosis of the underlying malignancy. We report a rare case of PNS associated with transitional cell carcinoma of the bladder in a 70-year-old male with a 6-month history of rapidly progressive symmetric sensory neuropathy. Peripheral neuropathy serological workup was unremarkable. A paraneoplastic neuropathy panel revealed anti-Hu autoantibodies. Further evaluation with a whole-body PET scan could not identify the primary malignancy, but it showed hypermetabolic hilar lymph nodes. An endobronchial ultrasound biopsy of the hilar lymph nodes was negative for cancer. The patient developed painless hematuria 2.5 years after the onset of the sensory neuropathy. Cystoscopy with biopsy revealed non-muscle-invasive transitional cell carcinoma of the bladder. Progression of the sensory neuropathy stopped after tumor resection. This case highlights the importance of a diligent and systematic approach to diagnose PNS. A relentless search is often required to detect PNSassociated occult malignancies.

\section{Karger ${ }^{\prime \prime}$}




\section{Introduction}

Paraneoplastic neurological syndromes (PNS) are immune-mediated nervous system disorders with diverse clinical manifestations [1]. Anti-neuronal nuclear antibody (ANNA-1) is among the most common autoantibody associated with PNS. ANNA-1, also known as the anti-Hu antibody, is a polyclonal IgG antibody directed against intracellular antigens of both tumor and neural cells [1]. Anti-Hu autoantibody-associated PNS can manifest as sensory neuropathy, sensory neuronopathy, autonomic neuropathy, cerebellar ataxia, and limbic encephalitis [2-4]. Small-cell lung carcinoma (SCLC) is the most common cancer associated with anti-Hu antibody PNS $[2,5]$. In addition to SCLC, Hodgkin's lymphoma and malignancies of the prostate, cervix, ovaries, thymus gland, and breast are known to cause anti-Hu PNS [6].

Although rare, transitional cell carcinoma (TCC) of the bladder - the most common urological malignancy - is also reported to be associated with anti-Hu PNS [7, 8]. Male gender, age, tobacco smoking, exposure to aniline dyes, drugs (cyclophosphamide and phenacetin), and pelvic irradiation are the established risk factors [7]. The first case report on anti-Hu PNS associated with TCC of the bladder was published in 2012 [8]. To our knowledge, this is the second case of TCC-associated anti-Hu PNS.

\section{Case Report}

The patient was a 70-year-old right-handed Caucasian male with a past medical history of Hodgkin's stage IVB lymphoma, status post-chemotherapy, in remission since 2002, nicotine dependence (50 PPD), chronic obstructive pulmonary disease, alcohol use disorder, hypertension, and dyslipidemia. He was referred to the neurology clinic for the evaluation of rapidly worsening sensory loss and tingling in his feet and hands on both sides. Sensory symptoms started 6 months prior to the clinic visit. Numbness and tingling initially appeared in the feet and progressed to involve the hands after 2 months. He had endorsed drinking at least 5 beers daily for 25-30 years but denied recreational drug use. His family history was negative for neuropathies.

Neurological examination demonstrated stocking-glove pattern hypoesthesia (impaired vibration, proprioception, and temperature sensation) up to the level of the mid-legs and mid-forearms. Deep tendon reflexes were diminished in both the upper and lower extremities. Romberg sign was noted and wide-based cautious gait was identified on examination. The remaining neurological examination was unremarkable.

The results of routineperipheral neuropathy laboratory work, such as complete blood count, comprehensive metabolic panel, thyroid-stimulating hormone, vitamin $\mathrm{B}_{12}$, methylmalonic acid, hemoglobin $A_{1 c}$, 2-h glucose tolerance test, and serum immunoelectrophoresis, were within normal limits. Low-yield investigations including erythrocyte sedimentation rate, antinuclear antibody, hepatitis $B$ and $C$ panel, complement 3 and 4, cryoglobulins, $\mathrm{c}$ - and p-ANCA, and HIV were negative. Nerve conduction study showedelectrophysiological evidence of moderate-to-severe diffuse demyelinating sensory neuropathy. Brain MRI was negative for intracranial involvement.

The rapid progression of the sensory neuropathy and unremarkable routine neuropathy workup results prompted a systematic search into rare etiologies. Further testing with a paraneoplastic panel revealed anti-Hu autoantibodies. Among paraneoplastic autoantibodies, the anti-Hu antibody has a high predictive value for underlying malignancies [2]. Hence, a PET scan was ordered to identify occult malignancy. The whole-body PET scan showed hypermetabolic bilateral hilar adenopathy. An endobronchial ultrasound biopsy of the hilar lymph nodes was negative for cancerous cells. Chest CT did not reveal abnormalities concerning for 


\section{Case Reports in Oncology}

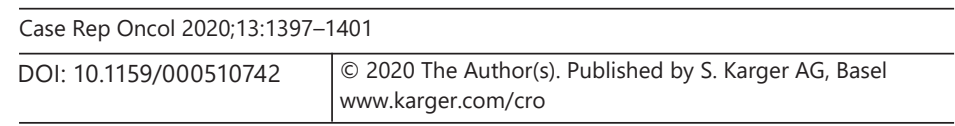

Ketineni et al.: Sensory Neuropathy Associated with TCC of the Bladder

Fig. 1. Polypoid mural-based nodular enhancing focus measuring $1.8 \times 1.6 \mathrm{~cm}$ along the left lateral urinary bladder wall.

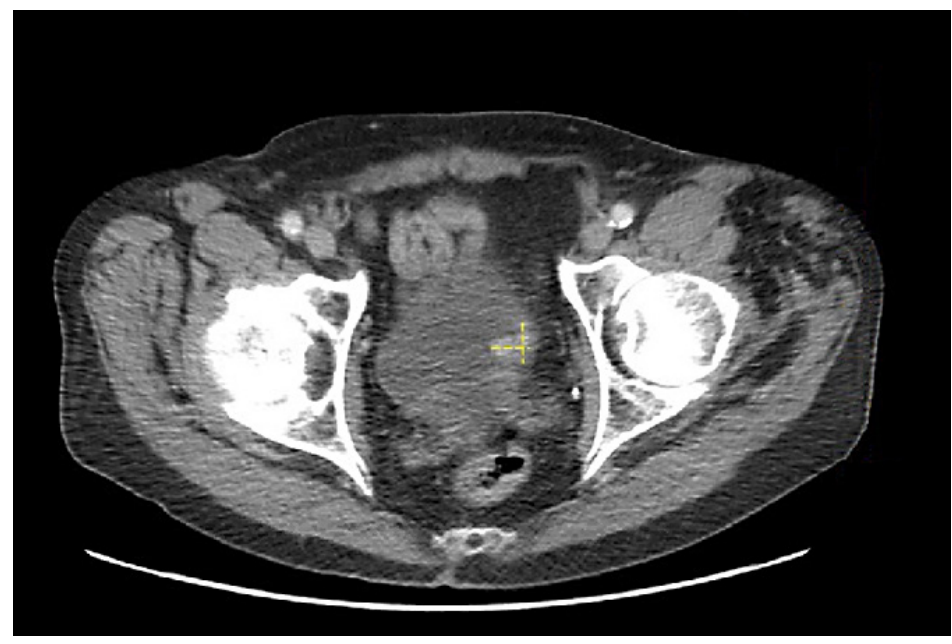

neoplasm. The ongoing progression of the sensory symptoms warranted treatment. A trial of intravenous immunoglobulin was attempted initially , but the patient developed a skin rash. A subsequent course of plasma exchange did not provide a substantial improvement in his symptoms.

He developed painless gross hematuria 2.5 years after the onset of the sensory neuropathy. Urine cytology revealed atypical cells suspicious for malignancy. A CT urogram demonstrated an irregular nodular mural wall-thickening with enhancement on the left side of the urinary bladder, as illustrated in Figure 1. Cystoscopy with subsequent transurethral resection of a bladder tumor showed three foci of high-grade papillary TCC. One focus was highly suspicious for lamina propria invasion without muscularis propria extension (G3 pT1N0M0). The patient underwent intravesicular mitomycin induction for 6 weeks, followed by a monthly maintenance dose for a year. A repeat cystoscopy after 1 year was negative for malignancy. The progression of the sensory neuropathic symptoms had stopped after tumor resection.

\section{Discussion}

We report the second case of anti-Hu PNS manifested by sensory neuropathy associated with TCC of the urinary bladder [8]. The first case of TCC (low-grade papillary carcinoma [G1 pTa] with grade 2 foci)-associated anti-Hu PNS was published in 2012; limbic encephalitis and sensory neuropathy were the presenting symptoms [8]. Our patient had exclusive sensory neuropathy without CNS involvement.

Diabetes mellitus, nutritional deficiencies, alcoholism, or toxin/medication exposure are some of the common causes of peripheral neuropathy in elderly patients. However, sensory neuropathy due to metabolic conditions typically has a gradual onset and progression. The hands are not usually effected early in the course of neuropathy due to metabolic conditions. Rapid worsening of sensory symptoms, along with early involvement of the hands, should raise suspicion for uncommon etiologies. A multidisciplinary approach is required to identify the underlying cancer in patients with PNS. An extensive search for the occult malignancy at the time of symptom onset is often futile, as PNS can precede a cancer diagnosis by up to 5 years $[9,10]$. Nevertheless, follow-up of PNS patients is crucial because a very high proportion of patients eventually develop a malignancy. Specifically, anti-Hu antibodies are associated with an $87 \%$ risk of developing SCCL or other tumors $[2,10]$. 
The pathophysiology of PNS is not entirely understood; autoantibodies and T-cellmediated immunity against onconeuronal antigens are widely accepted hypotheses $[9,10]$. These syndromes can involve the central and peripheral nervous systems, neuromuscular junctions, and muscles $[1,9,10]$. Amongst PNS, sensory neuropathy is the most common manifestation, accounting for approximately $50 \%$ of cases [2]. Sensory neuronopathy or ganglionopathy is a unique neurological syndrome associated with anti-Hu PNS, but an asymmetric presentation and involvement of the face and body differentiate it from more prevalent sensory neuropathy $[3,11,12]$. All patients with PNS should have age-appropriate cancer screening. Screening for colorectal and prostate cancer was negative in our patient. As stated in the Introduction, awareness of malignancies associated with anti-Hu autoantibodies (SCLC and prostate adenocarcinoma; breast, ovarian, gastrointestinal, and bladder malignancies; and Hodgkin's lymphoma) improves detection of the underlying cancer $[2,10]$. A 50-PPD smoking history increases the risk of malignancies, especially lung and bladder cancer $[7,13]$. A vigilant approach and knowledge of anti-Hu paraneoplastic syndromes helped our team to identify the bladder cancer promptly after the onset of hematuria. PNS secondary to antibodies against intracellular antigens (such as anti-Hu autoantibodies) does not respond well to immunosuppression or immunomodulatory interventions.

\section{Conclusions}

A high degree of clinical suspicion is required to diagnose uncommon PNS and their associated occult malignancies. A neurological consultation should be obtained in cases with atypical features such as rapid worsening of sensory symptoms and early involvement of the hands. Overall, anti-Hu paraneoplastic syndromes respond poorly to treatment. However, resection of the tumor may halt PNS progression.

\section{Statement of Ethics}

This case report was prepared and completed following the guidelines reported in the revised Helsinki Declaration of 2013. Written informed consent was obtained from the patient for the publication of the case report and accompanying images.

\section{Conflict of Interest Statement}

The authors have no conflicts of interest to declare.

\section{Funding Sources}

No targeted funding was obtained for this case report.

\section{Author Contributions}

S. Ketineni designed and conceptualized the case report, and prepared the initial draft of the manuscript. S. Gorantla designed, conceptualized, and finalized the manuscript. S. Kodali designed, conceptualized, and finalized the manuscript.

\section{Karger'}




\section{Case Reports in Oncology}

\begin{tabular}{l|l}
\hline Case Rep Oncol 2020;13:1397-1401 \\
\hline DOI: 10.1159/000510742 & $\begin{array}{l}\text { @ 2020 The Author(s). Published by S. Karger AG, Basel } \\
\text { www.karger.com/cro }\end{array}$ \\
\hline
\end{tabular}

Ketineni et al.: Sensory Neuropathy Associated with TCC of the Bladder

\section{References}

1 Galli J, Greenlee J. Paraneoplastic diseases of the central nervous system. F1000Res. 2020 Mar;9:F1000.

2 Graus F, Keime-Guibert F, Reñe R, Benyahia B, Ribalta T, Ascaso C, et al. Anti-Hu-associated paraneoplastic encephalomyelitis: analysis of 200 patients. Brain. 2001;124(Pt 6):1138-48.

3 Li J, Lin W. Various clinical features of patients with anti-Hu associated paraneoplastic neurological syndromes: an observational study. Medicine (Baltimore). 2018 May;97(18):e0649.

4 Senties-Madrid H, Vega-Boada F. Paraneoplastic syndromes associated with anti-Hu antibodies. Isr Med Assoc J. 2001;3(2):94-103.

5 Dalmau J, Furneaux HM, Rosenblum MK, Graus F, Posner JB. Detection of the anti-Hu antibody in specific regions of the nervous system and tumor from patients with paraneoplastic encephalomyelitis/sensory neuronopathy. Neurology. 1991;41(11):1757-64.

6 Ansari J, Nagabhushan N, Syed R, Bomanji J, Bacon CM, Lee SM. Small cell lung cancer associated with anti-Hu paraneoplastic sensory neuropathy and peripheral nerve microvasculitis: case report and literature review. Clin Oncol (R Coll Radiol). 2004;16(1):71-6.

7 Tanaka MF, Sonpavde G. Diagnosis and management of urothelial carcinoma of the bladder. Postgrad Med. 2011;123(3):43-55.

8 Lukacs S, Szabo N, Woodhams S. Rare association of anti-Hu antibody positive paraneoplastic neurological syndrome and transitional cell bladder carcinoma. Case Rep Urol. 2012;2012:724940.

9 Darnell RB, Posner JB. Paraneoplastic syndromes involving the nervous system. N Engl J Med. 2003;349(16): 1543-54.

10 Lancaster E. Paraneoplastic disorders. Continuum (Minneap Minn). 2015;21(2 Neuro-oncology):452-75.

11 Cowley A, Pascoe S. Paraneoplastic subacute sensory neuronopathy in association with adenocarcinoma of the prostate. BMJ Case Rep. 2011 Jul;2011:bcr0420114077.

12 Barohn RJ, Amato AA. Pattern-recognition approach to neuropathy and neuronopathy. Neurol Clin. 2013; 31(2):343-61.

13 Casey RG, Catto JW, Cheng L, Cookson MS, Herr H, Shariat S, et al. Diagnosis and management of urothelial carcinoma in situ of the lower urinary tract: a systematic review. Eur Urol. 2015;67(5):876-88. 